\title{
Virtual Crossing Numbers for Virtual Knots
}

\author{
Vassily Olegovich Manturov*
}

To the memory of my father

Oleg Vassilievich Manturov

(July,3,1936 - July, 23,2011).

\begin{abstract}
The aim of the present paper is to prove that the minimal number of virtual crossings for some families of virtual knots grows quadratically with respect to the minimal number of classical crossings.

All previously known estimates for virtual crossing number ([1, 3, 18, etc.) were principally no more than linear in the number of classical crossings (or, what is the same, in the number of edges of a virtual knot diagram) and no virtual knot was found with virtual crossing number greater than the classical crossing number.
\end{abstract}

MSC: 57M25, 57M27

Keywords: Knot, virtual knot, graph, crossing number, parity

\section{Introduction}

The main idea of the present paper is to use the parity arguments: if there is a smart way to distinguish between even and odd crossings of a virtual knot so that they behave nicely under Reidemeister moves then there is a way to reduce some problems about virtual knots to analogous problems about their diagrams (representatives).

Thus, we have to find a certain family of four-valent graph for which the crossing number (minimal number of additional crossings (prototypes of virtual crossings) for an immersion in $\mathbb{R}^{2}$ ) is quadratic with respect to the number of vertices (prototypes of classical crossings).

The study of parity has been first undertaken in [7, see also [8, 14] where functorial mappings from virtual knots to virtual knots were constructed, minimality theorems were proved, and many virtual knot invariants were refined. In the paper [15, by using parity, I constructed a diagrammatic projection mapping from virtual knots to classical knots.

In the case of graphs, such families having quadratic growth for the number of additional crossings with respect to the number of the crossings themselves are quite well known to graph theorists: even

\footnotetext{
*Peoples' Friendship University of Russia, vomanturov at yandex.ru

${ }^{\dagger}$ The author is partially supported by grants of RFBR 10-01-00748-a, RF President NSh - 3224.2010.1, Ministry of Education and Science of the Russian Federation 14.740.11.0794 and the Analytic Departmental Task Program "Development of the High School Scientific Potential"
} 
for trivalent graphs the generic crossing number grows quadratically with respect to the number of vertices, see, e.g., [17].

Notational remark. For graphs, we shall use the standard terminology: the number of vertices $v$, and the crossing number $c r$, the latter referring to the minimal number of additional crossings for generic immersions, see ahead. For virtual knots, we shall use the notation: $v i(K)$ and $\operatorname{cl}(K)$ for minimal virtual crossing number and minimal classical crossing number over all diagrams of a given knot.

\subsection{Acknowledgements}

I wish to express my special gratitude to D.P.Ilyutko, O.V.Manturov, L.H.Kauffman, S.Jablan, and A.V.Yudin for fruitful discussions.

I am especially grateful to Yu.S.Makarychev for fruitful consultations concerning graph theoretical questions.

\section{Virtual Knots and Crossing Numbers}

A virtual diagram is a four-valent graph on the plane where each crossing is either classical (in this case one pair of opposite edges are marked as an overcrossing pair, and the other pair is marked as an undercrossing pair; the undercrossing pair is drawn by means of a broken line) or virtual (virtual crossings are encircled).

Another way of looking at a virtual diagram is as follows. We say that a four-valent graph is framed if at every crossing of it, the four (half)edges incident to this crossings are split into two sets of (formally) opposite half-edges. An immersion of a four-valent graph in $\mathbb{R}^{2}$ is generic if all points having more than one preimage are intersection points of exactly two edges at their interior points. Then a virtual diagram is a generic immersion of a four-valent framed graph with all images of graph vertices endowed with classical crossing structure and all intersection points between edges marked as virtual crossings. Those points with more than one preimage are called crossing points.

A virtual link is an equivalence class of virtual diagrams modulo the detour move and the classical Reidemeister moves. Classical Reidemeister moves deal with classical crossings only; they refer to a domain of the plane with no virtual crossings inside. The detour move is the move which can be viewed as a transformation of the immersion outside the images of classical crossings: it takes an arc containing virtual crossings (and, possibly, self-crossings of an edge with itself) only and replaces it with an arc having the same ends but drawn in another way (all new crossings are to be virtual).

A virtual knot is a one-component virtual link. In this paper we deal with virtual knots only, however, the argument can be easily modified for the case of links.

The classical (resp., virtual) crossing number $\operatorname{cl}(K)$ (resp., vi $(K)$ ) of a virtual knot $K$ is the minimum of the numbers of classical (resp., virtual) crossings over all diagrams of $K$.

Classical crossing numbers of virtual knots were studied for a long time, see, e.g. [14, and references therein.

For estimates of virtual crossing numbers for virtual knots see [1, 3, 18. 
In the last years, some attempts to compare the classical and virtual crossing numbers were undertaken, e.g., Satoh and Tomiyama [18] proved that for any two positive numbers $m<n$ there is a virtual knot $K$ with minimal virtual crossing number $v i(K)=m$ and minimal classical crossing number $\operatorname{cl}(K)=n$.

However, no results were found in the opposite direction: for all known virtual knots the number of classical crossings was greater than or equal to the number of virtual crossings (see tables due to J.Green [5]).

In the present paper, we disprove this conjecture by reducing the problem from knots to graphs: we take some family of graphs for which $\mathrm{cr}$ grows quadratically with respect to the number of vertices, transform them into four-valent graphs (which can correspond to diagrams of virtual knots with classical vertices corresponding to crossings), turn these graph into a good shape (irreducibly odd, see ahead) by some transformations which increase the complexity a little, and then use the fact that for irreducibly odd graphs the crossing number is equal to the virtual crossing number of the underlying knots.

\section{$2.1 \quad 4-G r a p h s$ and Free Knots}

Now, let us change the point of view to virtual knots and consider some much simpler objects.

By a 4-graph we mean either a split sum of several 1-complexes each of which is either a regular finite 4-graph (loops and multiple edges are admitted) or is homeomorphic to a circle. By a vertex of a 4-graph we mean a vertex of some of its graph components. By an edge we mean either an edge of some of its graph components or a whole circle component. The latter are called circular edges.

All edges which are not circular are considered as equivalence classes of half-edges. We say that a 4-graph is framed if for each vertex of it, the four half-edges incident to this vertex are split into two pairs of (formally opposite) half-edges.

By a unicursal component of a framed 4-graph we mean either some of its circular components or an equivalence class of edges of some graph component, where the equivalence is defined as follows. Two edges $a, b$ are equivalent if there exists a chain of edges $a=a_{1}, \ldots, a_{n}=b$ for which each two adjacent edges $a_{i}, a_{i+1}$ have two half edges $a_{i}^{\prime}, a_{i+1}^{\prime}$ which are opposite at some vertex.

A framed 4-graph is oriented if all its circular components are oriented, and all edges of its graph components are oriented in such a way that at each vertex, for each pair of opposite edges, one of them is incoming, and the other one is emanating.

By a free link we mean an equivalence class of framed 4-graphs by the following equivalences (three Reidemeister moves):

The first Reidemeister move is an addition/removal of a loop, see Fig 1, left.

The second Reidemeister move adds/removes a bigon formed by a pair of edges which are adjacent in two edges, see Fig. 2, top.

Note that the second Reidemeister move adding two vertices does not impose any conditions on the edges it is applied to.

The third Reidemeister move is shown in Fig 3 top.

Note that these transformations may turn a circular component into a unicursal component of a framed 4-graph. 

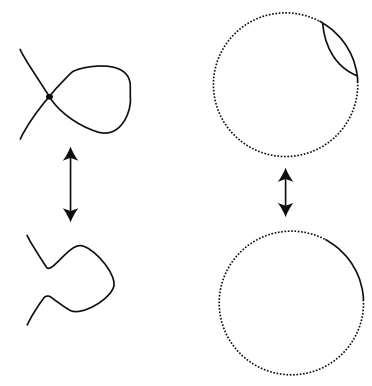

Figure 1: Addition/removal of a loop on a graph and on a chord diagram

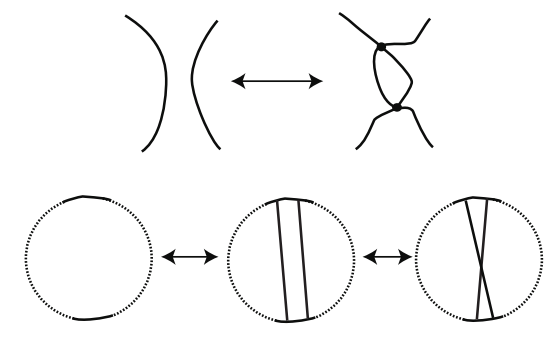

Figure 2: The second Reidemeister move and two chord diagram versions of it
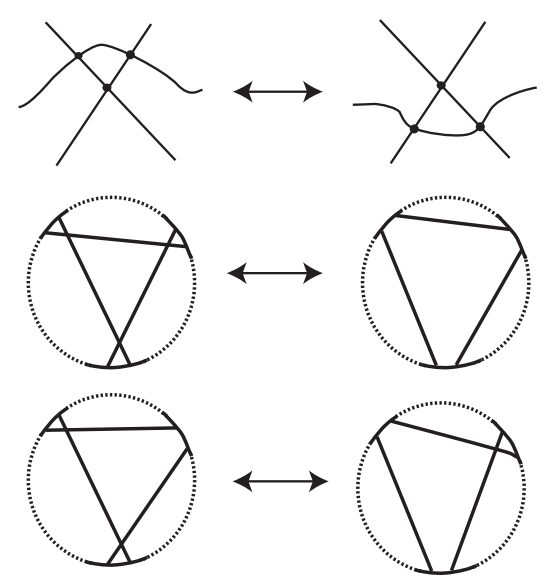

Figure 3: The third Reidemeister move and its chord diagram versions 


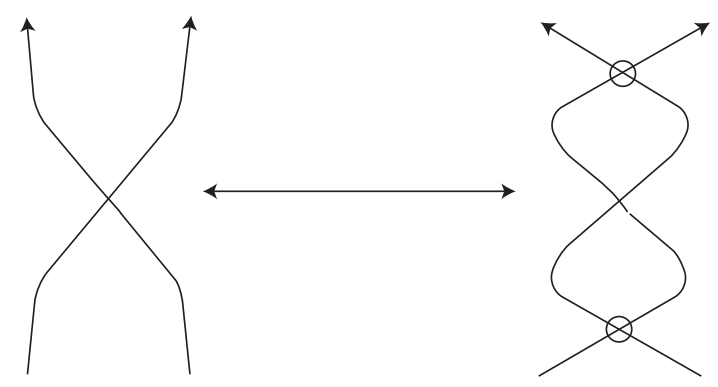

Figure 4: Virtualisation

The orientation of framed 4-graphs naturally leads to the notion of oriented free links.

One can easily see that the number of unicursal components of a framed 4-graph does not change under the Reidemeister moves. So, one can speak about the number of unicursal components of a free link. By a component of a link we mean an unicursal component unless specified otherwise.

A free knot is a 1-component free link.

Clearly, free knots and free links are equivalence classes of virtual knots and virtual links by the following two equivalences: the crossing switch $\longleftrightarrow \backslash$ and the virtualisation move; the latter move flanks a classical crossing by two virtual crossings, as shown in Fig. 4

The meaning of the first move is that we forget which branch of a knot is going over in a classical crossing (the other branch goes under); the meaning of the second move is that we allow to flip the cyclic clockwise (half)edge order at a crossing from $1,2,3,4$ to $1,4,3,2$.

\subsection{Chord diagrams}

A chord diagram is a finite cubic graph consisting of an cycle (the core) passing through all vertices and a collection of non-oriented edges connecting vertices. We also admit the empty chord diagram which is just a circle (in this case the circle is the core). A chord diagram is oriented if its core is oriented.

Chord diagrams are in one-to-one correspondence with framed 4-graphs having one unicursal component. We associate with the empty chord diagram the circle (the framed 4-graph with one component and no vertices) ; with any other chord diagram $D$ we associate the framed 4-graph as follows. We take the 1-complex obtained from the chord diagram $C$ as follows. Take the core $C o$ of the chord diagram $C$ and identify those points connected by chords; we get a 4-graph; for this 4-graph we say that two (half)-edges are opposite if they come from two (half)-edges approaching the same chord end on Co. Certainly, oriented chord diagrams are in a bijective correspondence with oriented framed 4-graphs with one unicursal component.

For chord diagrams, the Reidemeister moves look as shown in Fig 1, right 2 bottom 3 , centre and bottom.

We say that two chords $A, B$ of a chord diagram $C$ are linked if the two ends of the chord $B$ lie in distinct connected component of the complement to the endpoints of $A$ in the core circle of the Gauss diagram, and unlinked otherwise. For any chord $A$ we say that $A$ is unlinked with itself. We say that 


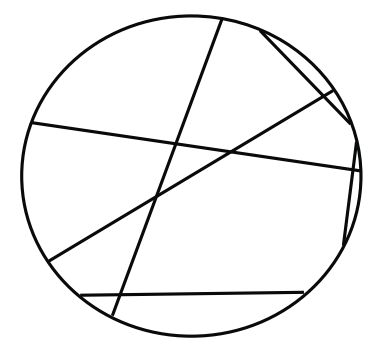

Figure 5: An irreducibly odd diagram

a chord $A$ of a chord diagram is even if it is linked with evenly many chords, and odd otherwise.

Analogously, for a framed 4-graph with one unicursal component we say that a crossing is even (resp., odd) iff the corresponding chord is even (resp., odd).

A chord diagram (resp., framed 4-graph with one unicursal component) is odd if all chords of it are odd. We say that an odd four-valent framed graph with one unicursal component is irreducibly odd if no second decreasing Reidemeister move can be applied to it. At the level of chord diagram this means that there are no two chords $A, B$ such that one end of $A$ is adjacent to one end of $B$ on the core circle, and the other end of $A$ is adjacent to the other end of $B$.

The importance of this notion is the following: the oddness of a framed 4-graph means that neither decreasing first Reidemeister move or a third Reidemeister move can be applied to it. The irreducible oddness also requires that no second Reidemeister move would be applicable. So, irreducibly odd framed 4-graphs can be operated on only by those Reidemeister moves which increase the number of crossings.

An irreducibly odd diagram is given in Fig. 5 .

As we shall see further, odd chords play a crucial role in the study of free knots.

\subsection{The bracket}

In the present section we shall introduce a simple invariant of free knots which allows one to reduce many problems about knots to problems about their representatives.

We shall start with the notion of smoothing. Let $\Gamma$ be a framed 4-graph. By smoothing of $\Gamma$ at $v$ we mean any of the two framed 4-graphs obtained by removing $v$ and repasting the edges as $a-b$, $c-d$ or as $a-d, b-c$, see Fig. 6. The rest of the graph (together with all framings at vertices except $v$ ) remains unchanged. We may then consider further smoothings of $\Gamma$ at several vertices.

Note that this operation may lead to circular connected components of the graph.

Let $\mathcal{G}$ be the set of equivalence classes of all four-valent framed graphs modulo the second Reidemeister move. Consider the formal $\mathbf{Z}_{2}$-linear space generated by all classes from $\mathcal{G}$.

Now, for a given framed 4-graph $\Gamma$, consider the following sum

$$
[\Gamma]=\sum_{\text {seven.,1 } 1 \text { comp }} \Gamma_{s}
$$




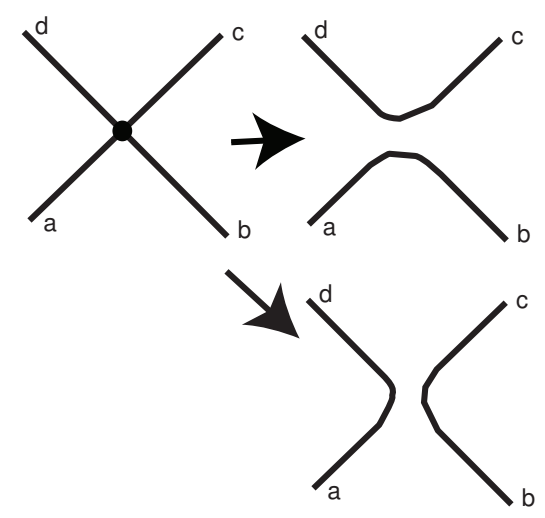

Figure 6: Two smoothings of a vertex of for a framed graph

which is taken over all smoothings in all even vertices, and only those summands are taken into account where $\Gamma_{s}$ has one unicursal component.

Thus, if $\Gamma$ has $k$ even vertices, then $[\Gamma]$ contains at most $2^{k}$ summands, and if all vertices of $\Gamma$ are odd, then we shall have exactly one summand, the graph $\Gamma$ itself. Consider $[\Gamma]$ as an element of $\mathbf{Z}_{2} \mathcal{G}$. In this case it is evident that if all vertices of $\Gamma$ are even then $[\Gamma]=\left[\Gamma_{0}\right]$ : by construction, all summands in the definition of $[\Gamma]$ are equal to $\left[\Gamma_{0}\right]$, it can be easily checked that the number of such summands is odd.

Now, we are ready to formulate the main result of the present section:

Theorem 1 ([]]). If $\Gamma$ and $\Gamma^{\prime}$ represent the same free knot then in $\mathbf{Z}_{2} \mathcal{G}$ the following equality holds: $[\Gamma]=\left[\Gamma^{\prime}\right]$.

Theorem 1 yields the following

Corollary 1. Let $\Gamma$ be an irreducibly odd framed 4-graph with one unicursal component. Then any representative $\Gamma^{\prime}$ of the free knot $K_{\Gamma}$, generated by $\Gamma$, has a smoothing $\tilde{\Gamma}$ equivalent to $\Gamma$ as a framed 4-graph. In particular, $\Gamma$ is a minimal representative of the free knot $K_{\Gamma}$ with respect to the number of vertices.

Indeed, if we look at an irreducibly odd graph $\Gamma$, we see that $\left[K_{\Gamma}\right]=\Gamma$. In the left hand side of this equality, $K_{\Gamma}$ means a free knot, i.e., an equivalence class of a 4-graph $\Gamma$ modulo the three Reidemeister moves. In the right hand side, we have just the graph $\Gamma$ modulo the second Reidemeister moves.

In fact, classification of elements from $\mathcal{G}$ is very easy.

Two graphs are equivalent whenever their two minimal representatives coincide.

So, in this case one can say that the bracket takes dynamical objects (framed 4-graphs modulo Reidemeister moves) to statical objects (framed 4-graphs modulo just the second Reidemeister moves, or just their minimal representatives).

In this way, in [7] I proved that free knots are generally not invertible: this was done by means of finding a good non-invertible representative for free links and some other orientation-sensitive parity arguments. 


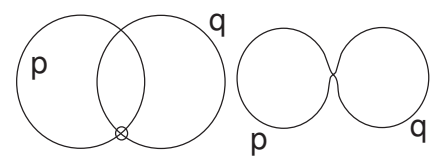

Figure 7: A 4-graph with two framings

\subsection{Crossing number for graphs}

Given a graph $\Gamma$; analogously to the case of four-valent graphs, by a generic immersion of $\Gamma$ in $\mathbb{R}^{2}$ we mean an immersion $\Gamma \rightarrow \mathbb{R}^{2}$ such that

1. the number of points with more than one preimage is finite;

2. tach such point has exactly two preimages;

3. these two preimages are interior points of edges of the graph, and the intersection of the images of edges at such a point is transverse.

By crossing number $\operatorname{cr}(K)$ of a graph $\Gamma$ we mean the minimal number of crossing points over all generic immersions $\Gamma \rightarrow \mathbb{R}^{2}$.

When we deal with framed 4-graphs, we restrict ourselves for such immersions for which at the image of every vertex the images of any two formally opposite edges turn out to be opposite on the plane.

Example 1. Consider the only 4-graph with one vertex $A$ and two edges $p, q$ connecting $A$ to $A$. There are two possible framings for this graph; one of these framings (where one half edge of $p$ is formally opposite to the other half edge of p) leads to a framed 4-graph with two unicursal components. Such a

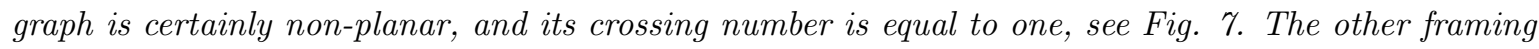
(where a half-edge of the edge $p$ is opposite to a half-edge of the edge $q$ ) is planar, so, for that framing the crossing number is 0 .

Now, let us present some examples of graphs where the crossing number grows quadratically. Let $p$ be a prime number; consider the chord diagram with $(p-3) / 2$ chords obtained as follows: take a standard circle $x^{2}+y^{2}=1$ on the plane, take all residue classes modulo $p$ except $0, p-1,1$, and put the residue class on the standard (core) circle as follows: the vertex corresponding to the residue class $r$ will be located at $\left(\cos \frac{2 \pi r}{p}, \sin \frac{2 \pi r}{p}\right)$. Now, every crossing $r$ is coupled with the crossing $s$ where $r s \cong 1 \bmod p$.

It is known that for such graphs for $p \rightarrow \infty$ the crossing number grows quadratically in $p$.

Other examples of families of trivalent graphs with quadratic growth can be constructed by using expander family; for more about expanders, see, e.g., [17. The idea is as follows: for a graph $\Gamma$ and a set $V$ of vertices of it, we define the neighbourhood $N(V)$ to be the set of vertices of $\Gamma$ not from $V$ which are connected to at least one vertex from $V$ by an edge. It is natural to study the ratio $\frac{|N(V)|}{|V|}$. A family $F_{n}$ of graphs is called an $\varepsilon$-expander family for some positive constant $\varepsilon$ if this ratio exceeds $\varepsilon$ for all graphs $F_{n}$ for sufficiently large $n$ and for all sets $V_{n}$ of vertices smaller than the half of all vertices of $F_{n}$. 


\section{The Main Theorem}

We are now ready to state and to prove our main result.

Theorem 2. For some infinite set of positive integers $i$, there is a family $V_{i}$ of virtual knots such that the virtual crossing number of $V_{i}$ grows quadratically with respect to the classical crossing number of $V_{i}$ as $i$ tends to the infinity.

The proof of this theorem relies upon the following lemmas.

Lemma 1. Let $K$ be a framed 4-graph. Let $K^{\prime}$ be a graph obtained from $K$ by smoothings at some vertices. Then $\operatorname{cr}\left(K^{\prime}\right) \leq \operatorname{cr}(K)$.

Lemma 2. Let $L_{n}$ be a family of trivalent graphs such that the crossing number $c r\left(L_{n}\right)$ grows quadratically with respect to the number of vertices $v\left(L_{n}\right)$ as $n$ tends to the infinity. Then there are two families of framed 4-graphs $\Gamma_{n}^{\prime} \Gamma_{n}$ such that

1. $\Gamma_{n}$ are all irreducibly odd;

2. The number of vertices of $\Gamma_{n}$ does not exceed 3 times the number of vertices of $L_{n}$.

3. $\Gamma_{n}^{\prime}$ is obtained from $\Gamma_{n}$ by smoothing of some vertices; both $\Gamma_{n}$ and $\Gamma_{n}^{\prime}$ are graphs with one unicursal component;

4. $L_{n}$ is a subgraph of $\Gamma_{n}^{\prime}$ obtained by removing some edges.

Proof of the Main Theorem. Let us take a family of trivalent graphs $L_{n}$ with quadratical growths of the crossing number. Denote their numbers of vertices by $v_{n}$ and denote their crossing numbers by $c r_{n}$.

Apply Lemma 2. Consider the families of graphs $\Gamma_{n}$ and $\Gamma_{n}^{\prime}$. Consider an arbitrary immersion of $\Gamma_{n}$ in $\mathbb{R}^{2}$. Endow all vertices of this immersion with any classical crossing structure; denote the obtained virtual knot by $K_{n}$.

We claim that the classical crossing number $\operatorname{cl}\left(K_{n}\right)$ of the knot $K_{n}$ grows linearly with respect to $c r_{n}$, whence the virtual crossing number $v i\left(K_{n}\right)$ grows quadratically with respect to $c r_{n}$. The first claim follows from the construction: the number of classical crossings of $K_{n}$ does not exceed tree times the number of vertices of $L_{n}$, so, the minimal classical crossing number over all diagrams representing the knot given by $K_{n}$ can be only smaller.

Now, consider $v i\left(K_{n}\right)$. Let $L$ be a diagram of the knot represented by $K_{n}$. So, if we consider the framed 4-graphs corresponding to diagrams $L$ and $K_{n}$, they will represent the same free knot. By definition, $K_{n}$ corresponds to the framed 4-graph $\Gamma_{n}$. Denote the framed 4-graph corresponding to $L$ by $\Delta$. We see that $\Delta$ represent the same free knot as $\Gamma_{n}$. Now, apply Theorem 1 to the free knot generated by $\Gamma_{n}$. By construction, it is irreducibly odd. Thus, we see that $\Gamma_{n}$ can be obtained from $\Delta$ by means of a smoothing at some vertices.

So, by Lemma 1, the (virtual) crossing number of $\Delta$ is bounded from below by the (virtual) crossing number $v i\left(K_{n}\right)$ of $K_{n}$. By definition, vi $\left(K_{n}\right)$, in turn, is bounded from below by the crossing number 


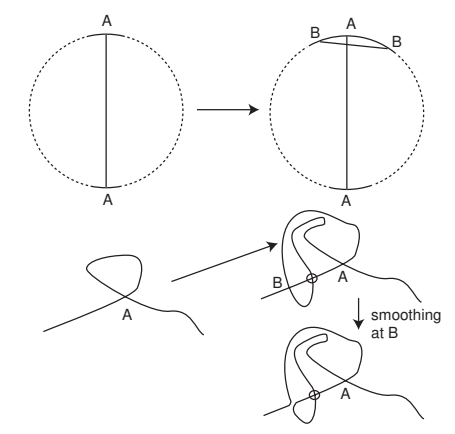

Figure 8: Addition of a chord and the inverse operation

of $\Gamma_{n}$. By Lemma 1 the latter is estimated from below by $\Gamma_{n}^{\prime}$. Finally, $\operatorname{cr}\left(\Gamma^{\prime}(n)\right) \geq \operatorname{cr}\left(L_{n}\right)$ because $L_{n}$ is a subgraph of $\Gamma_{n}$, and $\operatorname{cr}\left(L_{n}\right)$ grows quadratically with respect to the number of vertices of $L_{n}$.

This completes the proof of the Main Theorem.

Now let us prove auxiliary Lemmas 1 and 2 .

Proof of Lemma 1. Indeed, consider an immersion of $K^{\prime}$ in $\mathbb{R}^{2}$ preserving the framing and realising the crossing number $\operatorname{cr}\left(K^{\prime}\right)$. Now, take those vertices of $K$ where the smoothing $K^{\prime} \rightarrow K$ takes place and perform this smoothing just on the plane.

Proof of Lemma Q Let $L_{n}$ be a connected trivalent graph. Obviously, $n$ is even; let us couple the vertices of $L_{n}$ arbitrarily and connect coupled vertices by edges. We get a four-valent graph. We shall denote it by $\Gamma_{n}^{\prime}$; to complete the construction of $\Gamma_{n}^{\prime}$, we have to find a framing for it in order to get a diagram of a free knot (with one unicrusal component).

To do this, we shall use Euler's theorem that for every connected graph with all vertices of even valency there exists a circuit which passes once through every edge. Let us choose this circuit to be the unicursal circuit for $\Gamma_{n}^{\prime}$ thus defining the framing at each vertices (two consequent edges at every vertex are decreed to be formally opposite).

Consider the chord diagram of $\Gamma_{n}^{\prime}$. This diagram might well have even and odd chords. Our goal is to construct the chord diagram of $\Gamma_{n}$ by adding some chords to $\Gamma_{n}^{\prime}$. Namely, for every chord $l$ of $\Gamma_{n}$ we shall either do nothing, or add one small chord at one end of $l$ (linked only with $l$ ) or add two small chords at both ends of $l$. Our goal is to show that the obtain an irreducibly odd chord diagram such that the framed 4-graph of $\Gamma_{n}^{\prime}$ is obtained from the framed 4-graph of $\Gamma_{n}$ by smoothing of some vertices.

Note that whenever a chord diagram $Y$ is obtained from a chord diagram $X$ by adding one chord linked precisely with one chord of $X$ then the corresponding 4-graph of can be obtained from the framed 4-graph of $X$ by smoothing of some vertices.

Indeed, view Fig. 8

Without loss of generality we may assume that the chord diagram for $\Gamma_{n}^{\prime}$ has no solitary chords (chords not linked with any other chord). 
Now, to every odd chord of $\Gamma_{n}^{\prime}$ we add one small chord on one end of it. To every even chord of $\Gamma_{n}^{\prime}$ we add two chords on both flanks. This will guarantee that the resulting chord diagram is odd (all small chords are odd since each of them is linked with exactly one chord). Besides, this guarantees that the resulting chord diagram (or framed 4-graph) is irreducible.

We shall distinguish between former chords (belonging to $\Gamma_{n}^{\prime}$ and new chords (small added chords).

Now, no two former chords (for $\Gamma_{n}^{\prime}$ ) can be operated on by a second decreasing Reidemeister move: for each two chords of such sort $a, b$ there is at least one chord $c$ distinct from $a, b$ which is linked with $a$ and not with $b$ (it suffices to take one of the two small chords linked with $a$ ). A former chord can not participate in a second Reidemeister move together with a new chord because every former chord is linked with at least one former chord and at least one new chord it is linked with, and every new chord is linked with exactly one chord.

If two new chords $x$ and $y$ are linked with different former chords, they can not participate in the second Reidemeister move; neither they can if they are linked with the same former chord: in this case, since the former chord (say, $z$ ) is not solitary, there is at least one chord $w$ lying in between $x$ and $y$, so, the endpoints of $x$ and $y$ can not be adjacent.

Now, an obvious estimate shows that the number of chords of $\Gamma_{n}$ does not exceed $3 n$.

Remark 1. In this direction, one can prove a bit more than stated in the main theorem: the number of virtual crossings grows quadratically with respect to the number of classical crossings not only for virtual knots, but also for virtual knots considered modulo virtualisation.

\section{References}

[1] D. M.Afanasiev, (2010) Refining the invariants of virtual knots by using parity, Sbornik Mathematics, 201:6, pp. 3-18.

[2] M. Chrisman, V.O.Manturov, (2010) Combinatorial Formulae for Finite-Type Invariants via Parities, arXiv:math.GT/1002.0539

[3] H.A.Dye, L.H.Kauffman, (2010), Virtual Crossing Numbers and the Arrow Polynomial, In:Proceedings of the Conference, The Mathematics of Knots. Theory and Applications. M.Banagl, D. Vogel, Eds., Springer-Verlag.

[4] R. Fenn, L. H.Kauffman, and V.O. Manturov (2005), Virtual knot theory - unsolved problems, Fundamenta Mathematicae, 188, pp. 293-323

[5] J.Green, Virtual knot tables, http://www.math.toronto.edu/ drorbn/Students/GreenJ/

[6] F. Jaeger, L. H. Kauffman, and H. Saleur (1994), The Conway Polynomial in $S^{3}$ and Thickened Surfaces: A new Determinant Formulation, J. Combin. Theory. Ser. B 61, P. 237-259.

[7] V. O. Manturov, (2010), Parity in Knot Theory, Sbornik Mathematics, N.201, 5, P.65-110. 
[8] V.O. Manturov, Parity and Cobordisms of Free Knots, Sbornik Mathematics, to appear V. O. Manturov, Parity and See also: arXiv:math.GT/1001.2728.

[9] V. O. Manturov, V.O. (2004), Long virtual knots and Its invariants, Journal of Knot Theory and Its Ramifications, 13 (8), pp.1029-1039.

[10] V. O. Manturov, Free Knots and Parity (2011), arXiv:math.GT/09125348, v.1., to appear in: Proceedings of the Advanced Summer School on Knot Theory, Trieste, Series of Knots and Everything, World Scientific.

[11] L. H. Kauffman (1999), Virtual knot theory, European Journal of Combinatorics 20:7 , P. 662 690.

[12] L. H. Kauffman, V. O. Manturov (2006), Virtual knots and links, Proceedings of the Steklov Mathematical Institute, 252, P. 104-121.

[13] V. O. Manturov (2005), Teoriya Uzlov (Knot Theory, in Russian), M.-Izhevsk., RCD, 2005, 512 pp.

[14] V. O. Manturov (2010), Virtual'nye Uzly. Sovremennoe sostoyanie teorii (Virtual Knots: The State of the Art, in Russian), M.-Izhevsk., RCD, 490 pp.

[15] V. O. Manturov (2011), A Functorial Map from Virtual Knots to Classical Knots and Generalisations of Parity, arXiv:math.GT/1011.4640

[16] V. O., Manturov (2005), The proof of Vassiliev's conjecure on planarity of singular links, Izvestiya Mathematics: 69:5, pp. 169-178.

[17] J.Pach, F.Shakhrokhi, M.Szegedy, Applications of The Crossing Number, Algorithmica, Vol.16, 1, P.111-117.

[18] S.Satoh, Y.Tomiyama (2011), On the crossing number of a virtual knot, Proceedings of the AMS, Published Electronically, May 2011 